\title{
HuskyADAPT: A Project-Based Accessible Design Course (Experience)
}

\section{Dr. Dianne Grayce Hendricks, University of Washington}

Dr. Dianne Hendricks is a Lecturer in the Department of Human Centered Design \& Engineering and the Director of the Engineering Communication Program at the University of Washington. She designs and teaches courses involving universal design, technical communication, ethics, and diversity, equity and inclusion. She co-founded HuskyADAPT (Accessible Design and Play Technology), where she mentors UW students in design for local needs experts with disabilities. She also leads STEM outreach activities for the UW community and local K-12 students involving toy adaptation for children with disabilities. Dianne holds a PhD in Genetics from Duke University, and BS in Molecular Biology and BA in Psychology from the University of Texas at Austin.

\section{Dr. Anat Caspi P.E., The Taskar Center for Accessible Technology}

Dr. Anat Caspi is director of the Taskar Center for Accessible Technology housed by the Paul G. Allen School for Computer Science and Engineering at the University of Washington. Caspi received her PhD from the Joint Program in BioEngineering at University of California, Berkeley \& UCSF. Her research interests are in the areas of ubiquitous computing and data science. Caspi is interested in ways by which collaborative commons and cooperation can challenge and transform computing disciplines, and in particular, translation and deployment of technology to benefit individuals with disabilities.

\section{Dr. Heather A Feldner, University of Washington}

Heather Feldner is an Assistant Professor in the Department of Rehabilitation Medicine at the University of Washington. She received her BS in Human Biology, Master's degree in Physical Therapy from Marquette University, $\mathrm{PhD}$ in Disability Studies from the University of Illinois at Chicago, and completed a postdoctoral research position in the Department of Mechanical Engineering at the University of Washington. Heather has a special interest in user-centered design and participatory research, and has been a lab member of the GoBabyGo program, which creates custom safety and accessibility modifications to commercially available battery powered toy ride-on cars for children with disabilities, since 2012. Heather's research focuses on investigating the impact of traditional and alternative mobility technologies on the experiences of people with disabilities and their families, and the direct and indirect influences of physical and social environments, technology design, industry, and disability orientation on those experiences. She is a faculty advisor for the Husky ADAPT student organization and a co-instructor in the Husky ADAPT design course series.

\section{Ms. Molly Y. Mollica, University of Washington}

Molly Mollica earned her BS in Biomedical Engineering and her MS in Mechanical Engineering from Ohio State University. She is currently a PhD student in the Department of Bioengineering at the University of Washington. Her engineering education research focuses are in service learning, increasing diversity in engineering, and adapting toys for children with diverse abilities. Her bioengineering research focuses are in thrombosis mechanics, mechanobiology, and DNA origami nanotechnology.

\section{Shawn M Rundell, University of Washington}

Shawn M. Rundell, PT, DPT is a pediatric physical therapist and clinical teaching therapist at the University of Washington in the Division of Physical Therapy. She has experience working with individuals with a wide variety of neurological diagnoses across their lifespan and feels strongly that everyone should have access to toys, mobility and their environment to enhance their play skills and social interactions.

Mr. George Zatloka, Design Research Consultant Jennifer Mankoff, University of Washington 
Jennifer Mankoff is the Richard E. Ladner Professor in the Paul G. Allen School of Computer Science \& Engineering at the University of Washington. Her research focuses on accessibility, health and inclusion. Her work combines a combination of critical thinking and technological innovation. She strives to bring both structural and personal perspectives to her work. For example, her recent work in the intersection of mental health and discrimination uses sensed data to explore how external risks and pressures interact with people's responses to challenging moments. Similarly, her work in fabrication of accessible technologies considers not only innovative tools that can enable individual makers but also the larger clinical and sociological challenges to disseminating and sharing designs. Jennifer received her $\mathrm{PhD}$ at Georgia Tech, advised by Gregory Abowd and Scott Hudson, and her B.A. from Oberlin College.

\section{Dr. Katherine M Steele, University of Washington}

Dr. Steele is an associate professor in mechanical engineering at the University of Washington. She received her BS in engineering from the Colorado School of Mines and MS and $\mathrm{PhD}$ in mechanical engineering from Stanford University. She is the head of the Ability Lab, dedicated to designing new tools and techniques to improve human ability through engineering, and also a leader of AccessEngineering to enable individuals with disabilities to pursue careers in engineering. Dr. Steele previously worked in multiple hospitals as an engineer, including The Children's Hospital of Colorado, Lucille Packard Children's Hospital, and the Rehabilitation Institute of Chicago. 


\section{HuskyADAPT: A Project-Based Accessible Design Course (Experience)}

\section{Introduction}

In this Experience Report, we document the design and implementation of a project-based accessible design course offered by HuskyADAPT (Accessible Design and Play Technology) [1], a multidisciplinary initiative at University of Washington (UW) that promotes an inclusive culture and provides opportunities for students to engage in accessible design projects. The innovation of the course is the participatory design component with local people with disabilities, who serve as needs experts for the projects [2].

Here we document our experience in an inclusive design course where student teams work on projects centered on empowerment, independence, and community participation while promoting student engagement with local community members with disabilities and their allies as codesigners and needs experts. At the end of the course, we evaluated the impact of the course through student self-reported data and instructor observations. The course builds on lessons learned from our initial work in educational outreach and community engagement [3-6].

Our experience is relevant to engineering educators who aim to advance the participation of underrepresented populations in engineering, including people with disabilities, because:

1. Our course is built on a participatory design framework in all stages of the engineering design process that engages local needs experts, who are people with disabilities and organizations that support people with disabilities.

2. Through this course, we provide an example of an inclusive culture in engineering.

3. The HuskyADAPT course is an example of integrating accessibility into a design course, which aligns with the recent addition of accessibility in the ABET definition of engineering design [7].

4. The service learning component of our course may be especially attractive to underrepresented students in engineering, as engineering courses that include service learning commonly enroll a higher number of underrepresented students [8-12], and engineering classes or projects with clear service components commonly attract students from underrepresented groups [13-14].

5. Our course is focused on the positive social impact of engineering, which makes engineering more accessible to underrepresented students, as the potential to make a positive societal impact is especially important to underrepresented students in career selection [15-16].

\section{HuskyADAPT: Accessible Design and Play Technology}

HuskyADAPT was co-founded in 2016 by University of Washington (UW) faculty and students from Bioengineering, Computer Science, Human Centered Design and Engineering, Mechanical Engineering, and Rehabilitation Medicine [1]. HuskyADAPT has grown to include a student organization with over 100 active members. Our initial work was focused on educational outreach and community engagement involving a brief activity in accessible design: electronic toy adaptation for children with disabilities [2-6]. Since 2016, we have engaged over 1000 middle, high school and UW students in educational outreach events, and we have donated over 500 adapted toys to families of children with disabilities. The HuskyADAPT student 
organization holds two weekend events per month where volunteers adapt toys and families pick up adapted toys in a "drive-through" on campus.

Student and community member participants in toy adaptation outreach events reported:

1. Toy adaptation is enjoyable.

2. Learning about universal design through toy adaptation promotes awareness that engineering can have a direct impact on people.

3. Engaging in accessible design projects deepens participants' connection to the field of engineering.

Motivated in part by the overwhelmingly positive response we received to our outreach efforts, the HuskyADAPT student organization also accepts requests for adapted toys and accessible design projects from the local community through our website.

\section{Accessible Design Course: Overview}

Since 2017, several of the HuskyADAPT co-founders have offered a project-based course [6] that provides further opportunities for students to engage in accessible design. One innovative aspect of the course is the high level of involvement of local needs experts, who are people with disabilities or organizations that support people with disabilities, and the focus on a participatory design approach, which involves the end-users in every stage of the engineering design process. In other words, projects are co-designed for people with disabilities, by people with disabilities.

Each of the first two offerings of the two-quarter HuskyADAPT accessible design course had an enrollment of approximately 20-25 undergraduate and graduate students, and at least $65 \%$ of students were engineering majors. In addition to design journals and weekly reflections, assignments include team presentations in class and a poster at the end-of-quarter inclusive design showcase, where needs experts and the public also attend.

The projects we select for the accessible design course (1) can be completed in two 10-week quarters, (2) do not require specialized or advanced engineering expertise, and (3) involve needs experts who are interested in working with students and understand that the goal of the project is for students to gain experience rather than to deliver a completed project. (See Table II below.)

The HuskyADAPT course is an example of integrating accessibility into a design course, which aligns with the recent addition of accessibility in the ABET definition of engineering design [7]. The course learning objectives are:

1. Contribute and communicate effectively with a multidisciplinary team

2. Follow best practices of human-centered design

3. Identify, formulate, and solve design problems

4. Design a system, component, or process to meet desired needs within realistic constraints

5. Describe introductory concepts of disability studies and how they relate to engineering practice

6. Engage in and evaluate the co-design process with community members with disabilities

7. Identify the principles of inclusive design and how they benefit diverse communities

8. Devise an action plan to promote inclusivity and accessibility in engineering practice 
Course topics include: (1) disability studies, (2) universal design, (3) participatory design, (4) the human centered design process, and (5) prototyping. (See Table I describing course content and syllabus in Appendix A.)

\begin{tabular}{|l|l|}
\hline \multicolumn{2}{|l|}{ Table I. HuskyADAPT Accessible Design Course Topics and Assignments } \\
\hline Topic & Major Assignment \\
\hline Introduction to Disability Studies & Team Contract \\
\hline Define Your Challenge & \\
\hline Start Your Design & \\
\hline Design Requirements & \\
\hline $\begin{array}{l}\text { Guest Lecture: } \\
\text { Participatory Design with People with Disabilities }\end{array}$ & Design Feedback \\
\hline Ideation & Search-Discuss-Discover \\
\hline Presentations & Presentation \\
\hline Prototyping 101: Sketching, cardboard, foam, paper, clay & Low-Fi Prototype \\
\hline Guest Lecture: Design in Action & Prototype \\
\hline Design Decisions Prototypes & Design Feedback \\
\hline Design Aesthetics & Poster Draft \\
\hline Inclusive Design Showcase & Design Reflection \\
\hline
\end{tabular}

\section{Service Learning}

The course is focused on service learning, a powerful educational framework for delivering engineering curricula [8-10] that benefits student retention, personal development, and community connections [11-12]. Engineering classes or projects with clear service components commonly attract students from underrepresented groups [13-14], and making a positive impact in society is especially important to underrepresented students when choosing a career [15-16].

\section{Inclusive Engineering and Universal Design}

People with visible and invisible disabilities are an underrepresented minority in engineering education and careers. Our course aims to make students more aware of the need for inclusive engineering practices and universal design, which is the creation of products, processes, and spaces that are accessible to people with diverse abilities.

\section{Participatory Design}

In our course, each team of 3-6 students works with a local needs expert (person with a disability or an organization that supports people with disabilities) on a single project for two quarters. The students meet with the needs expert to identify needs and learn about current solutions. The students request feedback on iterations of their design, and the needs experts test the final prototypes. To support effective communication with needs experts, we emphasize to the students the importance of listening and using inclusive language (see Appendix B: Expectations for Working with Collaborators and Need Experts). 


\section{Examples of Accessible Design Projects}

Here we describe three example projects: Climbing Higher, e-NABLE Device and Research, and Wild Thing Challenge and show examples of student-built prototypes (Fig. 1). To support prototyping and best practices, students have access to a makerspace and are mentored by faculty and an independent design consultant.

- Climbing Higher. Accessible climbing wall for adults with cognitive disabilities, in collaboration with Outdoors for All, a national organization that provides accessible recreational activities [17]. The team designed thermochromatic holds that change color when held by a climber in order to track progress, facilitate communication, and motivate climbers.

- e-NABLE Device and Research. The device team created a wearable sensor to track hand motion with a 3D-printed partial palm prosthetic designed by e-NABLE, a global community of volunteer prosthetic makers [18]. The research team created a user experience study that aims to improve the design and usability of assistive technology; recruited individuals for interviews on their experiences with prosthetics, including users of the e-NABLE prosthetic hand, users of other limb prosthetics, and prosthetics designers.

- Wild Thing Challenge. In collaboration with a young boy who is currently learning how to use head-array switches, which are activated by tilting his head to one side and can be mounted in the headrest of a wheelchair, the team designed a new use head-array sensor to control the WildThing, a ride-on toy car. Other adaptations to the Wild Thing can be found at GoBabyGo [19].

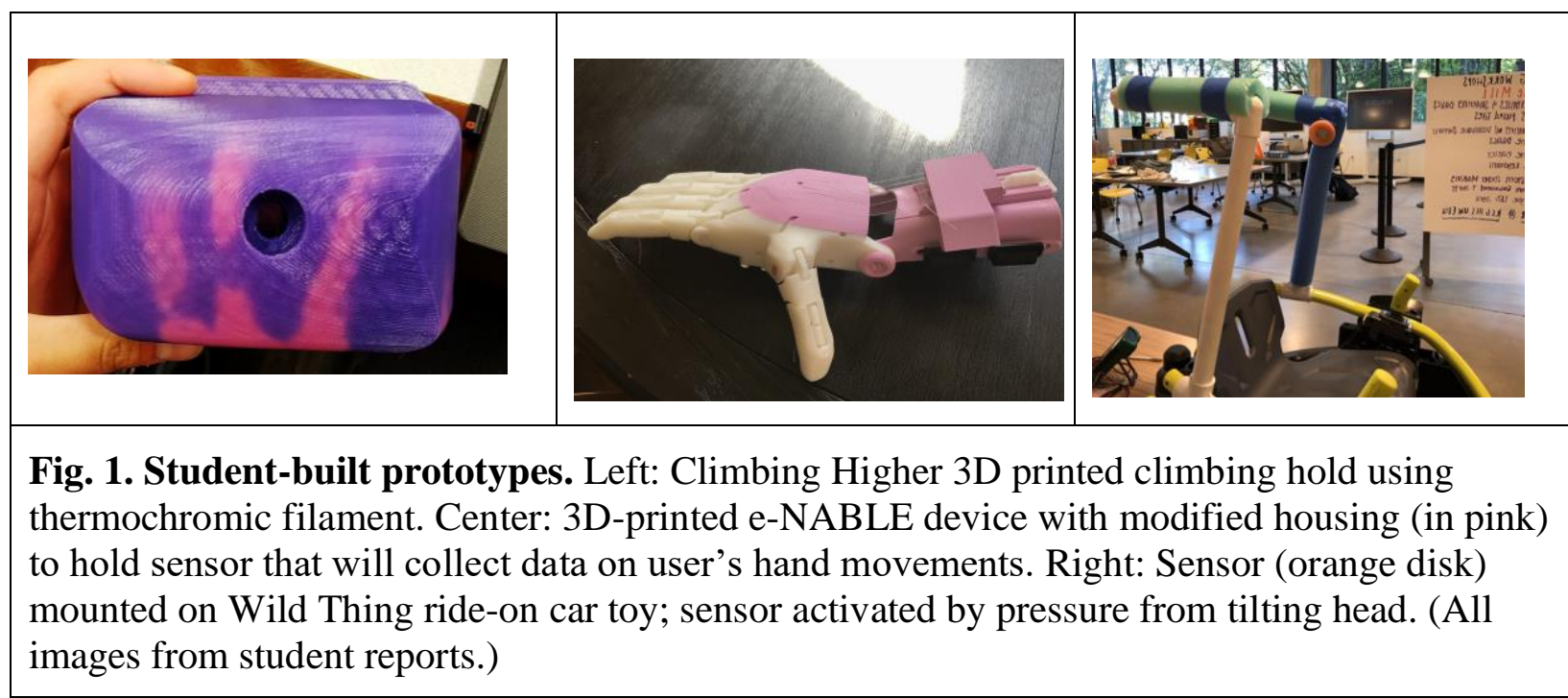

\section{Course Assessment}

Our course assessment methods are qualitative, and we are using this feedback for course improvement. At the end of the course, students were asked to reflect on what they enjoyed most, what they learned, and what they can use in the future. Students reported that they enjoyed the hands-on nature of the course, collaborating with the needs experts, working in interdisciplinary teams, and making a positive impact. (See Table II for excerpts of student reflections.) 
In addition, instructors received informal feedback from students during and after the course. Students routinely describe the HuskyADAPT course as "life-changing" and "the best class I've ever taken." Students easily identify something they learned that will serve them in their educational or career paths. We observe that the class not only satisfies students' desire for hands-on, project-based learning but also gives students opportunities to develop the ability to work in a team to accomplish self-assigned responsibilities; give and receive peer critique; and develop empathy. A common reason for student interest in the course is motivation to make a positive contribution to society.

\section{Table II. Representative comments from student reflections on HuskyADAPT course}

“...it was wonderful to work on a project that individuals have a real need for. Many of my design experiences have involved hypothetical end products that don't necessarily benefit its potential users."

"I really enjoyed the difficulty of the challenges presented to me in this project. Most engineering challenges are very deterministic and straight forward, whereas this project required some real in depth consideration of the problem and the actual person using the design."

"Designing and building my team's [solution] took a lot of different engineering skills, many of which my team and I had no experience with when we came into the project... Gaining this experience improved our confidence as engineers and helped prepare us to tackle unfamiliar challenges in the future."

"I learned so much about inclusivity in design. It made me more aware of design choices already made in my daily facilities and it made me question if these design choices are inclusive of everyone."

"In future [projects], I hope to talk with direct stakeholders in the earliest possible stage of the project. In addition, I can ensure that stakeholders are involved in the full design process so that their voices are heard. Participatory design is not only good for the scope of the project, but also the success of the final prototype developed."

"I also loved getting to learn how to talk about disabilities (i.e. people first language), because [this] will be forever useful throughout my life."

\section{Lessons Learned}

Our teams encountered a few challenges involving team dynamics and student communication issues, which was due in most cases to incompatible schedules and also different student priorities. We tried to keep updated on any student issues through weekly check-in sessions with instructors and a team agreement that each team created at the beginning of the quarter. 
Both the students and needs experts have been mostly positive about their co-designing efforts through the participatory design process. In the cases where the process has been challenging, the problems usually involved difficulty in finding a time for student teams and needs experts to meet face-to-face. Very few needs experts are available to travel to campus regularly, in part due to difficulties in arranging accessible transportation. In addition, some teams were disappointed that they worked with an organization instead of an individual. For example, as described above the Climbing Higher team worked with Outdoors for All; the students interacted primarily with a knowledgeable and experienced staff member who had worked with adults with cognitive disabilities at adaptive rock climbing events.

\section{Conclusion}

In this Experience Report, we describe the design, implementation, and impact of a novel curriculum in accessible design. The HuskyADAPT inclusive design course provides a collaborative, multidisciplinary opportunities for students to engage in accessible design projects that are co-designed with the end-users: local people with disabilities and organizations that support people with disabilities.

We hope that our work may serve as a resource. Here we have highlighted the important considerations for launching a project-based accessible design course, and to support faculty at other institutions we have provided a syllabus (Appendix A) and included recommendations to students for talking about disability (Appendix B). For more information on universal design, please see [20].

\section{References}

[01] HuskyADAPT: Accessible Design and Play Technology. https://depts.washington.edu/adaptuw/

[02] HuskyADAPT Vertically Integrated Projects course info. https://depts.washington.edu/adaptuw/design/design-projects-new/

[03] M. Mollica, H Feldner, A Caspi, KM Steele, D.G. Hendricks, “Toy Adaptation: an Initial Investigation Into Participant Experience and Perceptions", Pacific Northwest Section Conference, American Society for Engineering Education, Seattle WA. (2017)

[04] M. Mollica, H. Feldner, A. Caspi, K. Steele, and D.G. Hendricks. "Work in Progress: Toy Adaptation in Bioengineering Outreach to Demonstrate Societal Impact and Increase Diversity in Bioengineering." American Society for Engineering Education Annual Conference, Columbus, OH. (2017)

[05] M. Mollica, H. Feldner, A. Caspi, K. Steele, S. Israel, and D.G. Hendricks. "Toy Adaptation for Recruitment of Underrepresented Students to Bioengineering." American Society for Engineering Education Annual Conference, Salt Lake City, UT. (2018)

[06] M.Y. Mollica, A.M. Spomer, B.M. Goodwin, S. Israel, A. Caspi, H.A. Feldner, K.M. Steele, and D.G. Hendricks. "Engagement in Practice: Toy Adaptation for Children with Disabilities: Engaging the Community through Educational Outreach 
and Toy Donation”, American Society for Engineering Education Annual Conference, Tampa, FL. (2019)

[07] ABET Accreditation Criteria. https://www.abet.org/accreditation/accreditationcriteria/criteria-for-accrediting-engineering-programs-2018-2019/\#GC3

[08] J. Mankoff. "Practical Service Learning Issues in HCI", Association for Computing Machinery CHI Extended Abstracts on Human Factors in Computing Systems, Montreal, CA (2006).

[09] Oakes, W., Duffy, J., Jacobius, T., Linos, P., Lord, S., Schultz, W. W., \& Smith, A. "Service-learning in engineering". 32nd Annual Frontiers in Education, Boston, MA. (2002)

[10] Duffy, J., Tsang, E., \& Lord, S. Service-learning in engineering: What why and how? ASEE Annual Conference, St. Louis, MO. (2000).

[11] Eyler, J., \& Giles Jr, D. E. (1999). Where's the Learning in Service-Learning? Jossey-Bass Higher and Adult Education Series.

[12] Sax, L. J., Astin, A. W., \& Avalos, J. (1999). Long-term effects of volunteerism during the undergraduate years. The review of higher education, 22(2), 187-202.

[13] R. E. Davis, S. Krishnan, T. L. Nilsson and P. F. Rimland, "IDEAS Interdisciplinary Design Engineering and Service International Journal for Service Learning in Engineering, Humanitarian Engineering, and Social Entrepreneurship," pp. 165-179, 2014.

[14] C. Rader, D. Hakkarinen, B. M. Moskai and K. Hellman, "Exploring the appeal of socially relevant computing: are students interested in socially relevant problems?," Proceedings of the 42nd ACM Technical Symposium on Computer Science Education, pp. 423-42.( 2011)

[15] National Academy of Engineering. "Changing the Conversation: Messages for Improving Public Understanding of Engineering," The National Academies Press, Washington, DC, 2008.

[16] Google. "Women Who Choose Computer Science - What Really Matters: The Critical Role of Encouragement and Exposure," 2014.

[17] Outdoors for All. https://outdoorsforall.org/

[18] E-NABLE. http://enablingthefuture.org/

[19] GoBabyGo. https://www.gbgconnect.com/

[20] "What is Universal Design?" UW DO-IT. https://www.washington.edu/doit/whatuniversal-design-0 


\section{Appendix A. Abbreviated Syllabus: HuskyADAPT Accessible Design Course}

\section{PURPOSE}

Design projects centered on empowerment, independence, and community participation that engage local community members with disabilities and their allies as co-designers and needs experts.

\section{OBJECTIVES}

By the end of this course students should demonstrate their ability to:

1. Contribute and communicate effectively with a multidisciplinary team

2. Follow best practices of human-centered design

3. Identify, formulate, and solve design problems

4. Design a system, component, or process to meet desired needs within realistic constraints Describe introductory concepts of disability studies and how they relate to engineering practice

5. Engage in and evaluate the co-design process with community members with disabilities

6. Identify the principles of inclusive design and how they benefit diverse communities

7. Devise an action plan to promote inclusivity and accessibility in engineering practice

\section{PHILOSOPHY: STUDENT-LED DESIGN \& INNOVATION}

Each design team is responsible for setting their schedule and plan of action for each quarter. Make sure you set aside the time in your schedule to dedicate to this project, your team, and your needs expert. In our experience, going deep on a project provides more learning and rewards than working on a large number of projects. Use this experience to really test and develop your need-finding, design, fabrication, teamwork, and communication skills.

These projects will go as far as YOU decide to take them. The teaching team and the UW community are here to offer as much support and encouragement as possible towards your project. Please let us know how we can best support you to find the resources required to tackle your design challenge.

\section{VERTICALLY-INTEGRATED PROJECT (VIP)}

This course operates in conjunction with the University of Washington VIP Program, which supports hands-on, project-based, research and exploration. The VIP Program operates in a research and development context, with teams of students, faculty, and community members working on real-world projects. Students that participate in VIP earn academic credit for their participation in design efforts.

\section{ASSIGNMENTS}

Weekly Summary. These summaries are designed for you to have an opportunity to reflect and track your progress through the quarter. Each week you will need to submit your AOIs - a bulleted list with your Accomplishments, Objectives, and Issues from the week - and a picture. The picture can be of your design notebook, an interview, a prototype - anything that you are proud/excited/frustrated about from your week! 
Presentations. Presentations are your opportunity to share your progress and get detailed feedback. Detailed guidelines and a grading rubric for each presentation are posted on our course website.

Design Showcase. The HuskyADAPT Design Showcase will be a celebration of what you and your peers have accomplished this year! We invite the local community and serve food so that everyone can mingle, provide feedback, and try hands-on demonstrations with your design. You will create a poster to highlight your final design, as well as a table or space for hands-on demonstration and collecting feedback. We encourage you to create engaging experiences so that you can use this as an opportunity to get broad feedback on your design solution from the diverse audience of engineers, designers, and community members who join for this event.

Documentation. A big goal of this program is to share what you have done - so that your designs and hard work can live- on beyond this course. Documentation is often a way to amplify your impact! Designs can more easily be brought to mature stages with good documentation that is shared. Spring quarter you will work with the teaching team to define the best documentation to share your design and support future innovation.

Peer Evaluations. Students will review and be reviewed by their teammates through a peer review process. All students are required to perform peer evaluations at mid-quarter and the end of the quarter. Failure to complete the peer evaluations will result in a full letter grade deduction. Late peer review submissions will not be accepted. The peer evaluations will focus on contribution, communication, and leadership.

Design Reflection. At the end of each quarter, a 2-page report documenting your process and experience. This is an opportunity for you to look back at your design notebook, review your notes and prototypes, and carefully reflect on your growth and your team's progress. Some questions to consider: What worked or didn't work? How have you grown or developed? What are your future goals related to this project and accessible design? How would you describe this experience in an interview? What can you do to improve your impact in this course and future design challenges? Pictures can be included to support your story. Use this as an opportunity to also practice your writing, editing, and formatting.

Design Notebook. All students are required to maintain a design notebook. Design notebooks are where you can track your notes, ideas, sketches - everything about your process. This record helps you track and make sure you do not lose critical parts of your process. These notebooks increase efficiency, longevity, and sustainability of design projects. 


\section{Appendix B. HuskyADAPT Expectations for Working with Collaborators and Need Experts}

\section{General etiquette and behavior:}

- Be respectful of collaborator's time - come prepared with an agenda and plan of action. Before the meeting, decide who from your team will (1) lead the conversation, and (2) take notes. Make sure you have a plan (e.g., shared google doc) to record all the notes and reflections after the meeting.

- Remember this should be a conversation and collaboration, not an interrogation. Use your skills to engage with your collaborators, elicit stories, and set a strong foundation for your work together.

- Remember that you are representing UW. When interacting with the public - and answering any media questions - please act accordingly. Your words and actions may be memorialized in media and/or create an impression on the people at the event.

- Please wear appropriate clothing. "Business casual" - avoid anything too flashy.

- Follow all safety rules at all times! Whatever the place and environment.

- If you aren't sure, ask someone for help.

- Follow-up with a thank you and any notes or action items from the meeting.

\section{Respectful and Appropriate Language:}

Be sure to use respectful and appropriate language. Even though we know your intentions are good, your wording makes a difference. Please think about your word choice.

- Avoid saying "normal" or "abnormal". We are all different, with different abilities!

- Please use people-first language.

\begin{tabular}{|l|l|}
\hline \multicolumn{2}{|l|}{ Examples of People-First Language } \\
\hline Instead of this... :/ & Say this please! :) \\
\hline child who can't use the normal switch & child who uses an accessible switch \\
\hline confined to a wheelchair & person who uses a wheelchair \\
\hline someone who is suffering from a disability & $\begin{array}{l}\text { person who has a disability } \\
\text { person with diverse abilities }\end{array}$ \\
\hline Unfortunately, kids with disabilities can't... & This person can do tasks with one hand, etc. \\
\hline Kids with disabilities need special help to... & $\begin{array}{l}\text { This kid uses } \\
\text { This child is using an accessible switch. } \\
\text { (no need to call attention or justify why child's } \\
\text { actions are "different") }\end{array}$ \\
\hline $\begin{array}{l}\text { Child with a disability can't operate normal } \\
\text { toys or do normal stuff }\end{array}$ & $\begin{array}{l}\text { This child uses an accessible switch to... } \\
\text { Using his/her unique abilities, this child can... }\end{array}$ \\
\hline
\end{tabular}

